\title{
Ações de saúde mental na atenção básica: caminho para ampliação da integralidade da atenção
}

\author{
M ental health in primary care: ways to reach an integral care
}

Oswaldo Yoshimi Tanaka ${ }^{1}$

Edith Lauridsen Ribeiro ${ }^{2}$

${ }^{1}$ FaculdadedeSaúde

Publica, USP. Av. Dr.

Arnaldo 715 , Cerqueira

César. 01246-904

São Paulo SP.

oytanaka@usp.br

${ }^{2}$ Secretaria M unicipal de

SaúdedeSão Paulo.
Abstract $A$ great challenge for the primary healthcare system, implemented by the strategy called the Family $\mathrm{H}$ ealth Program, is to incorporate actions for facing situations of violence and mental health problems. This study analyzed the care delivered to 411 children between five and eleven years of age in a primary care unit in the city of São Paulo. The clinical findings were compared to a standard inventory of symptoms ( $\mathrm{CBCL}$ ). In addition, semistructured interviews were held with pediatricians. The study shows low capacity of the pediatricians to recognize mental health problems in children. This is mainly due to deficiencies in their training and lack of possibilities for concrete intervention to face a complaint or diagnostic hypothesis. The reorganization process of primary care will need to provide specific technical support in mental health, incorporating more appropriate technologies for intervention such as a humanized approach and qualified listening. The inclusion of psychosocial aspects in the everyday practice of primary care will make it possible to broaden the concept of health and open way for an integrated approach to situations of violence related to children assisted by the primary care network of the Brazilian Health System.

Key words Primary care, Mental health in children, $\mathrm{H}$ ealth professional, Performance, $\mathrm{H}$ ealth policy
Resumo A atenção básica de saúde, impulsionada pela estratégia da saúde da família, tem o grande desafio de incorporar de ações de enfrentamento às si tuações de violência e problemas de saúde mental. 0 trabalho analisou a atenção prestada a 411 crianças de cinco a onze anos em uma UBS na cidade de São Paulo. Os dados clínicos foram comparados com um inventário desintomaspadronizados Child Behavior Checklist (CBCL). Entrevistas semi-estruturadas foram aplicadas complementarmente em pediatras. 0 trabalho mostra que os pediatras têm baixa capacidade de reconhecer problemas de saúde mental em crianças. 0 s principais fatores relacionados a este baixo desempenho foram: deficiência na formação, carência de possibilidade de atuação concreta frente à queixa ou hipótese diagnóstica. 0 processo de reorganização do trabalho na atenção básica deveria contemplar a oferta de apoio técnico específico em saúdemental, propiciando a incorporação detecnol ogias deintervenção mais apropriadas, como acoIhimento e escuta qualificada. A incorporação dos aspectos psicossociais na prática cotidiana da atenção básica possi bilitará a ampliação do conceito saúde-doença e abrirá caminho para a abordagem das situações de violência às crianças atendidas na rede básica de serviços de saúde do SUS.

Palavras-chave Atenção básica, Saúde mental infantil, Formação profissional, Políticas de saúde 
Introdução

0 conceito do processo saúde-doença tem evoluído, nas últimas décadas, do foco principal nas doenças e morte (aproximações negativas) para concepções mais vinculadas à qualidade de vida da população, de produção social da saúde, ou seja, uma aproximação positiva. Saúde é então resultado de um processo de produção social que expressa a qualidade devida deuma população, entendendo-se qualidade de vida como uma condição de existência dos homens no seu viver cotidiano, um "viver desimpedido", um modo de "andar a vida" prazeroso, seja individual seja coletivamente ${ }^{1}$. As várias teorias sobre a causalidade das doenças também acompanharam este processo de mudança, migrando de uma concepção monocausal para uma concepção ampliada, que leva em conta outras dimensões al ém da biológica, como estilos de vida, meio ambiente e sistema de saúde².

Para além das mudanças nas concepções, foi necessário introduzir novas propostas de práticas. Partindo do paradigma flexneriano, calcado na biologia e especialização, foi preciso migrar para uma nova forma de entendimento de produção de saúde, como um processo de produção social, a partir de determinantes e condicionantes sociais, econômicos, ideológicos e cognitivos. Este novo paradigma permite a ruptura dos limites do setor saúde, incorporando, desta forma, a interdisciplinaridade como campo de conhecimento ea intersetorialidade como campo de prática ${ }^{1}$.

Todas estas mudanças só se tornam efetivas se transformadas em práticas concretas. São estas que produzem impacto na qualidade de vida da população. D esde o início da implantação do SUS, algumas propostas de organização dos serviços e das práticas têm buscado dar conta destedesafio. Dentre estas propostas, vale ressaltar a estratégia da atenção primária à saúde (APS)/atenção básica (AB) e o Programa de Saúde da Família (PSF).

\section{Atenção básica em saúde}

A formulação da APS foi construída ao longo das quatro últimas décadas e apresentou entendimentos distintos ao longo deste tempo. A pesar da concepção de níveis de assistência já existir desde a primeira metade do século $X X$, foi na Conferência de Alma-Ata, realizada em 1978, que foram enunciados os princípios e diretrizes que deveriam reger esta nova proposta. No Brasil, a APS ganhou contornos específicos ${ }^{3}$. O Programa de Agentes Comunitários de Saúde (PACS) surgiu em 1991 e o
PSF, em 1994. Estes dois programas ganharam impulso rapidamente, principalmente com a publicação da Norma O peracional Básica - 01/964 (N OB 96) e do plano de metas para PSF em 1997. Com o tempo, este último tornou-se a estratégia estruturante dos sistemas municipais de saúde na busca da mudança do paradigma assistencial e da reorientação do modelo assistencial ${ }^{5}$.

A atenção básica deve ser a porta de entrada preferencial do SUS enível deatenção para atuação nas seguintes áreas estratégicas: eliminação da hanseníase, controle da tuberculose, controle da hiperten são e da diabetes mellitus, eliminação da desnutrição infantil, saúdeda criança, mulher eidoso, saúde bucal e promoção da saúde 5 . Com a posição estratégia no Sistema Ú nico de Saúde (SUS) de garantir a universalidade do acesso e a cobertura universal, cabeà atenção básica a efetivação de integralidade: integração de ações programáticas e demanda espontânea; articulação das ações de promoção à saúde, prevenção de agravos, vigilância à saúde, tratamento e reabilitação; trabalho de forma interdisciplinar e em equipe, ecoordenação do cuidado na rede de serviços ${ }^{5}$. A Estratégia da Saúde da Família (ESF) configura-se como principal modalidade de atuação da atenção básica. Seus princípios são: atuação no território através do diagnóstico situacional, enfrentamento dos problemas de saúde de maneira pactuada com a comunidade, buscando o cuidado dos indivíduos e das famílias ao longo do tempo; buscar a integração com instituições e organizações sociais e ser espaço de construção da cidadania ${ }^{5}$.

A pesar dos princípios enunciados acima apontarem para efetiva ampliação das ações para além do modelo biomédico, em especial aquelas relacionadas ao contexto social, não há, nos textos oficiais do M inistério da Saúde formatadores da atenção básica, referência explicita à incorporação de ações voltadas ao enfrentamento dos problemas de saúde mental.

\section{Políticas desaúde mental}

Desde os anos setenta e acompanhando a trajetória da reforma sanitária, o processo da reforma psiquiátrica vem alterando conceitos e práticas na atenção aos transtornos mentais no país. 0 foco fundamental deste movimento é a desinstitucionalização, sendo sua luta principal a redução do número de leitos nos manicômios e a implementação de ampla rede comunitária de serviços substitutivos ${ }^{6}$. Este direcionamento da reforma psiquiátrica para o cuidado dos pacientes com transtor- 
nos severos e persistentes e para a implantação de Centros de Atenção Psicossocial (CAPS) deixou em segundo plano a assistência aos transtornos mentais menos graves e mais prevalentes.

$\mathrm{Na}$ busca da redução desta defasagem na assistência, a Coordenação Geral da Saúde M ental (CGSM ) - DAPE/SAS/M S desenvolveu, a partir de 2001, uma série de documentos sobre a articulação entre a saúde mental e a atenção básica. As principais diretrizes para esta articulação são ${ }^{7}$ :

. A poio matricial de saúde mental às equipes de PSF: aumento da capacidade resolutiva das equipes;

- Priorização da saúde mental na formação das equipes da atenção básica;

- Ações de acompanhamento e avaliação das ações de saúde mental na atenção básica;

Uma das principais estratégias propostas é a criação de equipes de apoio matricial, cuja função consiste nas ações de supervisão, atendimento compartilhado e capacitação em serviço, realizado por uma equipe de saúde mental para equipes ou profissionais da atenção básica ${ }^{6}$.

Esta direcionalidade das políticas públicas de saúde mental visando à ampliação das ações na atenção básica é corroborada por diretrizes emanadas de organismos internacionais. A Organização M undial deSaúde(OM S), em 1990, publicou o documento "La introducción deun componentede salud mental en la atención primaria", no qual enfatiza a importância do aspecto emocional na atenção à saúde: "É impossível alcançar saúde se não se cuida das necessidades emocionais" e reforça que "as tarefas de saúde mental não são uma nova carga para os serviços de atenção primária; pelo contrário, aumentam a efetividade desta" ${ }^{8}$. Em 2001, a OMS reforça esta orientação quando publica dez recomendações para o enfrentamento dos problemas de saúde mental, sendo a primeira delas proporcionar tratamento na atenção primária9.

A atenção básica tem potencial para desenvolver dois principais tipos de ações de saúde mental. 0 primeiro consiste em detectar as queixas relativas ao sofrimento psíquico e prover uma escuta qualificada deste tipo de problemática; o segundo compreende as várias formas de lidar com os problemas detectados, oferecendo tratamento na própria atenção básica ou encaminhando os pacientes para serviços especializados. Goldberg e Huxley ${ }^{10}$ descreveram, em 1980, um esquema que permite compreender o caminho trilhado na busca de assistência no campo da saúde mental. São descritos cinco níveis, cada um representando uma população diferente de sujeitos. Para ir de um nível para outro, é necessário ultrapassar um filtro. Cumpre ressaltar que, dos quatro filtros descritos, dois estão na atenção básica (Quadro 1).

A pesar dos esforços da Coordenação Geral da SaúdeM ental/DAPE/SAS/M S, a inclusão efetiva de assistência à saúde mental na atenção básica ainda é uma realidade pouco freqüente. No documento "Política Nacional da Atenção Básica-2006"5, não há nenhuma referência às ações de saúde mental e não há indicadores relativos às ações de saúde mental realizadas pelas equipes do PSF no Sistema de Informação da Atenção Básica (SIAB), ainda que propostas já tenham sido elaboradas?

No ano de 2007, foi aprovada a inclusão da Saúde M ental como prioridadeno Pacto pela Vida (Portaria MS n³99/GM de 22/02/2006). Foram então elencados dois indicadores (Portaria MS $\mathrm{n}^{\circ}$ 325/GM de 13/03/2008): taxa de cobertura de CAPS/ 100.000 habitantes (principal) e taxa decobertura do Programa de Volta para Casa (complementar). Cabe destacar que, mesmo com estas importantes conquistas, ainda não há referência às ações e indicadores que contemplem a saúde mental na atenção básica.

\section{Saúde mental infantil na atenção básica}

$N$ a população infantil, são encontradas altas taxas de prevalência de transtornos mentais. Em revisão de literatura recente ${ }^{11}$, que incluiu trabalhos realizados a partir de 1980, foram encontradas taxas de prevalência global de transtornos mentais que variaram de 1,0\% a 51,0\% (média de 15,8\%). Estes estudos foram realizados em mais de vinte países e a faixa etária pesquisada variou de um a dezoito anos. As taxas de prevalência tendem a aumentar proporcionalmente com a idade, sendo que a pre-

Quadro 1. Acesso à atenção psiquiátrica.

nível 1: morbidade de base populacional

filtro 1: conduta do paciente e da família

nível 2: morbidade na atenção primária

filtro 2: detecção do transtorno - atenção básica

nível 3: morbidade psiquiátrica conspícua

filtro 3: encaminhamento para o psiquiatra - atenção básica nível 4: total de pacientes psiquiátricos

filtro 4: admissão ao hospital psiquiátrico - serviços

especializados

nível 5: pacientes psiquiátricos internados

Fonte: Goldberge H uxley ${ }^{10}$. 
valência média entre os pré-escolares foi de $10,2 \%$, entreos pré-adolescentes (seis a dozeou trezeanos), de $13,2 \%$, e entre os adolescentes, de $16,5 \%$. No Brasil, apesar dos poucos estudos publicados ${ }^{12}$, é provável que as taxas sejam semelhantes.

Vários trabal hos apontam para o pequeno reconhecimento ou valorização, pelos pediatras e/ ou outros profissionais da atenção básica, dos transtornos mentais na infância eadolescência. Em outra revisão de literatura ${ }^{13}$, abrangendo doze pesquisas sobre avaliação e tratamento dos transtornos mentais por pediatras, foi encontrada grande amplitude das taxas de diagnóstico, que variaram de $0,6 \%$ a $16,0 \%$. A maioria destas pesquisas encontrou taxas entre $4,0 \%$ e $7,0 \%$, aproximadamente metade daquelas encontradas pel os estudos de base populacional. Esta revisão também apresenta outros achados interessantes:

. Os pais não costumam queixar-se dos problemas emocionais ou de comportamento de seus filhos aos pediatras;

. Os pediatras têm dificuldade de identificar os problemas ou valorizar sua importância;

- Os pediatras têm receio de colocar "rótulos deletérios" em seus pequenos pacientes;

- Os pediatras são relutantes em expressar o diagnóstico para os pais por recear não ter tempo ou habilidade suficiente para lidar com o problema.

Costello et al. ${ }^{14}$, estudando 789 crianças de sete a onze anos atendidas por pediatras na atenção primária, encontraram taxa de prevalência de transtornos mentais de $22,0 \%$. N este mesmo estudo, a freqüência deste tipo de problema encontrada pelos pediatras foi de $5,6 \%$. N este mesmo estudo, os pediatras identificaram apenas $17,0 \%$ do total de crianças com problemas de saúde mental, levando a uma "new hidden morbidity" de 83,0\%, conforme os autores denominaram os falsos ne gativos. Dulcan et al. ${ }^{15}$, estudando os fatores parentais que influenciam o reconhecimento pelos pediatras dos problemas de saúde mental das crianças, encontraram queo nível de estresse dos pais, a história familiar de doença psiquiátrica e a discussão das preocupações dos pais com o pediatra são importantes para aumentar a capacidade de detecção destes problemas pelo médico.

Com o objetivo de contribuir para o aprimoramento e compreensão do processo de atenção no campo da saúde mental na infância, o presente estudo busca investigar a abordagem dos problemas de saúde mental (PSM ) durante atendimento na atenção básica ${ }^{16,17}$.

\section{Metodologia}

A pesquisa utilizou como estratégia o desenho de um estudo de caso descritivo e exploratório, com abordagem quantitativa e qualitativa. Foram estudadas 411 crianças de cinco a onze anos atendidas em uma Unidade Básica de Saúde (UBS) da cidade de São Paulo no ano de 2000.

Os dados analisados compreenderam: prontuário médico, aplicação de dois inventários sobre sintomas de problemas de saúde mental das crianças respondidos pelos pais ou responsáveis Strengths and Difficulties Questionnaire (SDQ) e Child Behavior Checklist ( $\mathrm{CBCL}$ ), questionário para os pais para a caracterização da família e entrevistas semi-estruturadas aplicadas aos médicos pediatras (onze profissionais). O SDQ, que é um questionário curto, foi aplicado após a consulta pediátrica para selecionar possíveis casos de PSM na clientel a atendida pela U BS eo CBCL foi utilizado na visita domiciliar para identificar casos com diagnóstico provável de transtorno mental.

Os eixos temáticos definidos para análise das entrevistas com os médicos foram:

. Concepção dos PSM ;

- Reconhecimento dos PSM ;

- Ação do pediatra frente aos PSM .

\section{Resultados}

Este trabalho apontou que os pediatras apresentam baixa sensibilidade para reconhecer problemas de saúde mental em crianças de cinco a onze anos atendidas em uma UBS da cidade de São Paulo. De modo geral, os pediatras têm alta especificidade, isto é, têm boa capacidade para distinguir os verdadeiros negativos. A reduzida capacidade de reconhecimento dos PSM pelos pediatras é semelhante à apontada na literatura, indicando dificuldade destes profissionais nessa área específica, mesmo considerando as diferenças de contexto social.

Para análise da capacidade de detecção dosPSM na atenção básica, foi utilizado $0 \mathrm{CBCL}^{18}$ para a identificação de casos com diagnóstico provável de transtorno mental. Os resultados da aplicação deste instrumento foram comparados com as hipóteses diagnósticas de PSM anotadas nos prontuários das crianças. A aplicação deste instrumento indicou que apenas $25,3 \%$ das crianças com escore clínico no $\mathrm{CBCL}$ e, portanto, com provável PSM , foram detectadas pelos pediatras. 
Quanto às hipóteses diagnósticas de PSM realizadas pelos pediatras, foi possível verificar que aquelas relacionadas à área dos transtornos específicos do desenvolvimento (linguagem, por exemplo) ou à área somática (enurese, bruxismo) são as hipóteses diagnósticas mais freqüentes. Por outro lado, hipóteses diagnósticas de transtornos ansiosos ou depressivos, que na literatura internacional correspondem a uma prevalência média, nesta faixa etária, de aproximadamente $10,0 \%{ }^{19}$, não foram feitas pelos médicos. Outro achado interessante foi que, em muitos casos, o pediatra identifica que algo não vai bem com a criança e aponta como hipótese diagnóstica apenas um aspecto do quadro clínico, isto é, acaba explicitando o diagnóstico, por exemplo, de enurese para uma criança com quadro clínico de ansiedade.

Quanto à preocupação dos pais em relação aos PSM dos filhos, foi encontrada associação positiva significativa entreescoreclínico do CBCL epreocupação dos pais, indicando boa sensibilidade dos pais em relação à presença de sintomas dePSM nos filhos. Por outro lado, a presença de preocupação dos pais não aumenta a capacidade de diagnóstico de PSM pelos pediatras, o que aponta para problemas na abordagem de questões do campo da saúdemental duranteo atendimento pediátrico. 0 processo da consulta médica, centrado em queixas a serem confirmadas pelo exame físico e/ou exames laboratoriais, acaba levando a uma desvalorização das queixas psicossociais que, apesar de verbalizadas no início da consulta, perdem sua potência e acabam ficando sem encaminhamentos adequados.

Quanto à conduta mais freqüente do pediatra anotada nos prontuários frente aos problemas detectados, foram identificados orientação $(23,6 \%)$ e encaminhamento para serviço de saúde mental e fonoaudiologia (15,3\%). A penas $1,7 \%$ do total das crianças com hipótese diagnóstica de transtorno mental recebia ou estava para receber assistência especializada em saúde mental. Também vale ressaltar que, dentre as 72 crianças com hipótese diagnóstica de PSM feita pelo pediatra, 21 (29,2\%) ficaram sem nenhum tipo de conduta registrada no prontuário, o que poderia estar indicando deficiência na formação profissional ou carências do próprio serviço.

A análise do material coletado a partir das entrevistas com os médicos pediatras que prestam assistência na UBS permitiu algumas considerações que possibilitam ampliar o entendimento da questão e apontou direções a serem seguidas em busca de uma assistência à saúde mental na atenção básica com maior qualidade.

No eixo temático sobre concepção dos PSM
(Quadro 2), foi claramente reconhecido o referencial teórico ampliado característico da pediatria, que inclui expressões como assistência integral, biopsicossocial e psicoprofilaxia. Também foi possível identificar um enfoque descritivo, que se aproxima das atuais formas de classificação dos transtornos mentais. Uma idéia marcante nas falas foi a concepção que implica o meio ambiente nas origens dos PSM das crianças, praticamente isentando-as, seja biológica ou psicologicamente, como co-participantes da gênese destas dificuldades.

No eixo temático sobre reconhecimento dos PSM (Quadro 3), aparece uma ampla gama de imagens. Não há clareza da magnitude dos PSM ; porém, entendendo quequando o problema égrave ele "chega aos ol hos da gente". Um importante núcleo de idéias relaciona-se com a incapacidade do profissional de acolher as questões de saúde mental, apesar das concepções ampliadas do atendimento em pediatria verbalizadas anteriormente. Os próprios médicos elencam as possíveis causas destas dificuldades, que passam pela linguagem hermética do campo da saúde mental (em especial a psicanálise), pelas características de personalidade do próprio profissional e, principalmente, por falhas na formação profissional. As deficiências estão presentes desde a formação básica (faculdade de medicina), em que os aspectos psicológicose emocionais da prática médica são relegados a um segundo plano. Por outro lado, as tecnologias de intervenção frente aos problemas detectados privilegiam medicamentos e intervenções "armadas", e valorizam pouco outros tipos de abordagens.

No eixo temático sobre ação dospediatrasfrente aos PSM (Q uadro 4), foi identificada uma dicotomia: o que épossível fazer e o que não é possível fazer na atenção básica. Os pediatras reconheceram que não dominam a área de saúde mental nem seus problemas e instrumental de intervenção com a mesma profundidade que outras especialidades da medicina. A dificuldade de se deparar com um problema que não sabem abordar ou não têm instrumentos claros de intervenção para lançar mão faz com que se mantenham longe destas questões, desval orizando-as na prática clínica diária. 0 desconhecimento de tecnologia apropriada imobiliza os pediatras, fazendo-os minimizar as queixas explícitas e não inquirir, durante a consulta, sobre questões de saúde mental.

Dentre as possibilidades de atuar dos pediatras foram encontrados aspectos contraditórios. Em muitas falas foram descritas situações de intervenções concretas que não são valorizadas pe los pediatras. Estas atuações são remeti das ao senso comum, ao bom senso de cada um, deixando 
Quadro 2. Esquema das dimensões do eixo temático Concepção de Problemas de Saúde M ental.

\begin{tabular}{|c|c|}
\hline Dimensão & Verbalizações \\
\hline Referencial teórico da pediatria & $\begin{array}{l}\text { Assistência integral } \\
\text { Atenção biopsicossocial } \\
\text { Influência do emocional no orgânico } \\
\text { Pediatria é desenvolvimento } \\
\text { Psicoprofilaxia } \\
\text { Entendimento da criança no seu meio } \\
\text { Coisas preventivas }\end{array}$ \\
\hline Descrição dos problemas de saúde mental & $\begin{array}{l}\text { Comportamento que chama atenção } \\
\text { Dificuldades de relacionamento } \\
\text { Sofrimento psíquico } \\
\text { Baixa auto-estima }\end{array}$ \\
\hline \multirow[t]{4}{*}{ Concepção de causalidade dos problemas de saúde mental } & $\begin{array}{l}\text { Escola } \\
\text { Fracasso escolar } \\
\text { Psicologização do fracasso escolar } \\
\text { A escola é uma porcaria } \\
\text { Dificuldade de valorização } \\
\text { Professor desmotivado }\end{array}$ \\
\hline & $\begin{array}{l}\text { Situação familiar } \\
\text { Família não tradicional - ausência de pai ou mãe } \\
\text { Drogas - alcoolismo } \\
\text { Falta de estímulo }\end{array}$ \\
\hline & $\begin{array}{l}\text { Problemas socioeconômicos } \\
\text { Privação social }\end{array}$ \\
\hline & $\begin{array}{l}\text { Multifatorial } \\
\text { Reflexo daquilo que acontece na família, na casa, } \\
\text { na comunidade }\end{array}$ \\
\hline
\end{tabular}

de serem vistas como procedimentos científicos, tecnologias de intervenção respaldadas pelo "saber oficial". Intervenções do tipo: "ouvir mais", "marcar consultas mais freqüentes", "dar mais atenção", "conversar com a criança”, "tentar conter", por não fazerem parte do arsenal terapêutico clássico dos pediatras, não são entendidos como científicos. Desta forma, eles acabam verbalizando que"criança é uma coisa ótima, acaba resolvendo sozinha", ou mesmo "quando a gente entra neste campo parece que a gente não é mais médico".

A organização do processo de trabalho dos médicos também foi apontada como obstáculo para a detecção destes problemas. Duas foram as principais justificativas levantadas: o curto tempo das consultas e a falta de profissionais especializados (ou equipes multiprofissionais) que pudessem servir de retaguarda no caso de dificuldades durante 0 atendimento. 0 encaminhamento para serviçosespecializados também é visto como algo problemático, difícil, com baixo retorno enão confiável.

Os resultados encontrados nesta pesquisa apontam que o planejamento das ações destinadas à melhoria da qualidade da assistência à saúde mental da infância na atenção básica deve incluir mudanças na formação profissional do médico, tanto na graduação como nos diversos tipos de pós-graduação (residência médica e especializações), que permitam otimizar sua capacidade de fazer diagnósticos precoces, realizar intervenções pertinentes à atenção básica e encaminhamentos oportunos e com co-responsabilização. 
Quadro 3. Esquema das dimensões do eixo temático Reconhecimento de Problemas de Saúde M ental.

\begin{tabular}{|c|c|}
\hline Dimensão & Verbalizações \\
\hline $\begin{array}{l}\text { M agnitude e gravidade } \\
\text { dos problemas de saúde mental }\end{array}$ & $\begin{array}{l}\text { Acho que tem...bastante } \\
\text { Eu acho que tem um mundo } \\
\text { Não sei se é muito, se é pouco } \\
\text { Dificilmente a gente pega uma coisa mais grave, muito } \\
\text { estruturada, psiquiátrica } \\
\text { Percentagem muito pequena com problemas psiquiátricos } \\
\text { O grave chega aos olhos da gente }\end{array}$ \\
\hline \multirow{3}{*}{$\begin{array}{l}\text { Dificuldade para identificar os } \\
\text { problemas de saúde mental }\end{array}$} & $\begin{array}{l}\text { A maior parte dos pediatras não lida } \\
\text { Falha na formação } \\
\text { Formação voltada para o orgânico } \\
\text { Não tem muito instrumental para estar detectando }\end{array}$ \\
\hline & $\begin{array}{l}\text { A gente acaba ignorando... } \\
\text { Depende da personalidade (do profissional) } \\
\text { Tem gente que não suporta nada do mental } \\
\text { Eu me sinto uma intrometida na vida da criança }\end{array}$ \\
\hline & $\begin{array}{l}\text { Não consigo fazer este diagnóstico } \\
\text { Linguagem é importante } \\
\text { Linguagem é inacessível } \\
\text { Linguagem atrapalha }\end{array}$ \\
\hline
\end{tabular}

Quadro 4. Esquema das dimensões do eixo temático Ação do Pediatra frente aos Problemas de Saúde Mental.

\begin{tabular}{|c|c|}
\hline Dimensão & Verbalizações \\
\hline Tecnologia de intervenção & $\begin{array}{l}\text { Consultas mais freqüentes, tentar conter, acaba resolvendo } \\
\text { sozinho } \\
\text { Vincular aquele paciente, não rotular imediatamente } \\
\text { Acaba escutando, dando atenção, criança é uma coisa ótima } \\
\text { Tentar trabalhar, falta subsídio teórico }\end{array}$ \\
\hline Dificuldades da intervenção & $\begin{array}{l}\text { É a experiência pessoal de cada um } \\
\text { Precisei do meu bom sensoFica como ansiedade nossa, você fica } \\
\text { meio perdido } \\
\text { Não tem o que fazer, não tem uma cesta básica para dar } \\
\text { Parte psicológica é relegada; não tem supervisão. Precisa atender } \\
\text { depressa, a equipe é muito pequena }\end{array}$ \\
\hline Organização do processo de trabalho & $\begin{array}{l}\text { Encaminha para psicólogo da rede } \\
\mathrm{N} \text { ão tem retorno do psicólogo } \\
\mathrm{O} \text { investigar do psicólogo é um investigar longínquo } \\
\mathrm{N} \text { ão que a higiene mental vá resolver, porque não existe mágica } \\
\mathrm{N} \text { ão encaminha porque não tem para onde encaminhar }\end{array}$ \\
\hline Preconceito & $\begin{array}{l}\text { M eu filho não é doido } \\
\text { Põe um caminhão na mãe } \\
\text { Um certo preconceito de estar atuando nesta área, porque o } \\
\text { pediatra acaba se achando menos responsável }\end{array}$ \\
\hline
\end{tabular}


Há também a necessidade de investir no aperfeiçoamento da organização do trabalho, tanto na unidade como na articulação entre as várias instâncias do sistema de saúde, visando a uma atenção em rede.

Da análise do estudo acima e a partir da realidade concreta dos serviços do SUS, é possível inferir pelo menos duas linhas principais de ações:

- Implantação efetiva de oferta concreta de serviços de apoio em saúde mental e sua articulação em rede. Fica claro na fala dos médicos que a falta de rede de suporteé componente importante, pois quando o profissional identifica questões de saúde mental muitas vezes não sabe 0 que fazer e para onde encaminhar;

- Capacitação dos profissionais da rede básica de saúde. 0 referido estudo mostrou que as atividades de educação permanente devem enfatizar, para além de questões conceituais, formas práticas de intervenção, pois os pediatras já têm, em grande parte, conceitos ampliados de saúde que envolvem aspectos psíquicos e sociais. A dificuldade principal étransformar em prática esta conceituação, tendo em vista a sua formação essencialmentebiomédica.

\section{Consideraçõesfinais}

$\mathrm{Na}$ busca da universalidade da assistência e da ampliação do acesso aos serviços de saúde, a imple mentação da atenção básica, através de sua estraté gia estruturante Saúde da Família, tem sido a principal diretriz das políticas públicas na direção da melhoria da qualidade da atenção à saúde no nosso país. Esta expansão da atenção básica exigiu a incorporação de recursos humanos não adequadamente preparados para este tipo de trabalho. Segundo documentos oficiais do M inistério da Saúde, o PSF deve dar conta de $85 \%$ das necessidades de saúde da população cadastrada, sendo que as questões psicossociais não estão incluídas como prioridades.

Pelo menos em tese, a proximidade da equipe da comunidade e a possibilidade de acompanhamento integral longitudinal permitiriam a abordagem de questões mais complexas do ponto de vista dos seus determinantes sociais. Dentre os problemas de saúde que emergem com a aproximação das comunidades, destacam-se os questões de saúde mental. Como apontado no estudo, esta "nova" demanda explicita as deficiências dos serviçostanto relativas à insuficiência na formação da equipe de saúde quanto à carência de instrumentos e apoio organizacional para a resolução e/ou encaminha- mento dos problemas identificados e/ou demandados pelos usuários. Assim os profissionais envolvidos na expansão da atenção básica, principalmente o médico, ancoram-se nos aspectos biológicos dos problemas de saúde, por serem estes os de maior familiaridade e capacidade de intervenção e que, em essência, foram mais afiançados na conformação da oferta de serviços de saúde, seja pelo financiamento ou pelas políticas explícitas das três esferas de governo na gestão do SUS.

As dificuldades en contradas na organização dos sistemas locais ou loco-regionais de saúde para acolhimento/captação dos usuários portadores de transtornos mentais são potencializadas pela "sensação" de incapacidade técnica de intervenção dos profissionais de saúde, principalmente 0 médico com formação clínica não especial izada, que constitui a maioria dos profissionais médicos incorporados na atenção básica. Assim, a proposta do M inistério da Saúde de implantação de apoio matricial para as equipes do PSF parece caminhar no sentido de subsidiar a ampliação da clínica e possibilitar a inclusão concreta e efetiva de ações de saúde mental na atenção básica. Cabe real çar que este apoio deverá ser ofertado a toda rede de atenção básica, pois o PSF cobre apenas uma parcela da população no país.

De modo análogo, outras demandas comuns na comunidade, quepoucas vezes aparecem no serviço de saúde, são aquelas relacionadas com situações de violência. Os problemas de saúde derivados dos distintos modos da violência surgem nos serviços de saúde de diferentes formas, al gumas claramente explícitas, outras nem tanto. Com freqüência são queixas fragmentadas, como "caiu e bateu a cabeça" ou "tem dificuldade para dormir", que não apontam, de forma imediata, a real dimensão e etiologia do problema. Muitas vezes fica mais fácil cuidar exclusivamente das lesões físicas, sem maiores envolvimentos com o contexto ou encaminhar crianças agitadas, insones ou que apresentam dificuldades na escola para tratamento psicológico, onde, com freqüência, permanecem longo tempo em filas de espera. A possibilidade de acolher as queixas relativas às situações de violência na sua complexidade depende da capacidade de trabal har com o conceito ampliado do processo saúde-doença e da competência e habilidade para lidar com questões para além do paradigma biomédico.

Ao olhar o usuário de manei ra abrangente, em suas dimensões física, mental e social, as equipes da atenção básica tornar-se-ão mais aptas ao enfrentamento das situações de violência. Acreditamos que, ao assumir a incorporação efetiva de ações de atenção à saúde mental como estratégia 
estruturante para ampliar o leque de problemas passíveis de resolução neste nível de atenção, estaremos contribuindo para um real avanço no redesenho do processo de trabal ho na atenção básica. A implementação de atividades de educação permanentedirigidas às equipes multiprofissionais da atenção básica possibilitará a incorporação efetiva de tecnologias leves, entendidas como acolhimento, vínculo e responsabilização, em contraponto às tecnologias leves-duras (saberes e práticas bem estruturados) e duras (máquinas e instrumentos ${ }^{20}$, para dar conta tanto da necessária ampliação da capacidade para deteç̧ão das situações de violência como da identificação de alternativas no âmbito das parcerias comunitárias que permitam a construção das redes sociais de apoio e o enfrentamento das situações de violência. Além disto, 0 caminho através da incorporação da atenção à saúde mental na rede básica necessita contar também com suporte de equipes atuantes e solidárias no repasse e intercâmbio de conhecimentos e tecnologias de intervenção para que todos os profissionais na rede básica possam lidar com as dificuldades emocionais que este enfrentamento traz.
A efetiva abordagem dos problemas de saúde mental pela equipe da atenção básica, incluído aí uma escuta qualificada e intervenções pertinentes neste nível de atenção, éum marcador potente que aponta a incorporação na prática cotidiana do conceito ampliado do processo saúde-doença. Dessa maneira, será possível potencializar a capacidade das equipes para sair da atuação tipo "queixa-conduta" e gerar competência para articular recursos comunitários e intersetoriais. 0 conhecimento do contexto sociocultural e dos recursos da comunidade e da família são condições necessárias para 0 enfrentamento de questões que extrapolam os problemas da ordem do biológico, como os transtornos mentais e aqueles derivados das situações de violência.

0 investimento na melhoria da atenção aos problemas de saúde mental é passo intermediário necessário e imprescindível para a incorporação da violência como objeto de intervenção dos serviços de saúde. É importante salientar que nenhuma outra situação de saúde apresenta este grau de complexidade, que contempla aspectos biológicos, emocionais e sociais.

\section{Colaboradores}

OY Tanaka e EL Ribeiro trabalharam na concepção, delineamento, análise, interpretação dos dados e redação do artigo. 


\section{Referências}

1. Mendes EV. Uma agenda para a saúde. $2^{a}$ ed. São Paulo: Hucitec; 1999.

2. Breilh J. Epidemiologia: economia, política e saúde. São Paulo: UNESP/H ucitec; 1991.

3. Gil CRR. Atenção primária, atenção básica e saúde da família: sinergias e singularidades do contexto brasileiro. Cad Saúde Pública 2006; 22(6):1171-1181.

4. Brasil. Ministério da Saúde. Norma Operacional Básica do Sistema Ú nico de Saúde/N OB-SUS 96. Brasília: Ministério da Saúde; 1997.

5. Brasil. M inistério da Saúde. Secretaria de Atenção à Saúde. Departamento de Atenção Básica. Política nacional de atenção básica. Brasília: M inistério da Saúde; 2006.

6. Brasil. M inistério da Saúde. Secretaria de Atenção à Saúde/DAPE. Saúde M ental no SUS: acesso ao tratamento e mudança do modelo da atenção. Relatório de Gestão 2003-2006. Brasília: Ministério da Saúde; 2007.

7. Brasil. Ministério da Saúde. Secretaria de Atenção à Saúde/DAPE-DAB. Saúde mental na atenção básica: o vínculo e o diálogo necessários [site na Internet]. [acessado 2007 out 8]. Disponível em: http://portal. saude.gov.br/portal/arquivos/pdf/diretrizes.pdf

8. Organização Mundial de Saúde. La introducción de un componente de salud mental en la atención primária. Genebra: OM S; 1990.

9. OPAS/OMS. Saúde mental: nova concepção, nova espe rança. Genebra: OMS; 2001.

10. Goldberg D, Huxley P. M ental illness in the community - the pathway to psychiatric care. London: Tavistock Publications Ltd; 1980.

11. Roberts ER, Attkisson CC, Rosenblatt A. Prevalence of psychopathology among children and adolescents. Am J Psychiatry 1998; 155:715-725.

12. Fleitlich-Bilyk B, Goodman R. Prevalence of child and adolescent psychiatric disorders in southeast Brazil. J Am Acad Child Adoles Psychiatry 2004; 43(6):727-734.
13. Costello EJ. Primary care pediatrics and child psychopathology: a review of diagnostic, treatment and referral practices. Pediatrics 1986; 78(6):1044-1051.

14. Costello EJ, Edelbrock C, Costello AJ, Dulcan M, Burns B, Brent D. Psychopathology in pediatric primary care: the new hidden morbidity. Pediatrics 1988; 82:415-422.

15. Dulcan M, Costello EJ, Costello AJ, Edelbrock C, Brent $\mathrm{D}$, Janiszewiski $\mathrm{S}$. The pediatrician as gatekeeper to mental health care for children: do parents' concerns open the gate? J Am Acad Child Adolesc Psychiatry 1990; 29:453-458.

16. Lauridsen-Ribeiro E, Tanaka OY. Problemas de saúde mental das crianças: abordagem na atenção básica. São Paulo: Annablume; 2005.

17. Tanaka OY, Lauridsen-Ribeiro E. Desafio para a atenção básica: incorporação da assistência em saúde mental. Cad Saúde Pública 2006; 22(9):1845-1853.

18. Bordin IAS, Mari JJ, Caeiro M F. Validação de versão brasileira do "Child Behavior Checklist" (CBCL) (Inventário de comportamentos da infância e adolescência): dados preliminares. Revista ABP-APAL 1995; 17:55-66.

19. Goodman R, Scott S. Child Psychiatry. Oxford: Blackwell Science; 1997.

20. Merhy EE. Em busca de ferramentas analisadoras das tecnologias em saúde: a informação e o dia a dia em um serviço, interrogando e gerindo trabalho em saúde. In: Merhy EE, Onoko R, organizadores. Agir em Saúde: um desafio para o público. 2ª ed. São Paulo: Hucitec; 2002. p. 113-150.

Artigo apresentado em 05/11/2007

Aprovado em 13/05/2008

Versão final apresentada em 07/10/2008 